\title{
A new method of subarachnoid puncture for clinical diagnosis and treatment: lateral atlanto-occipital space puncture
}

\author{
Dianrong Gong, BS, Haiyan Yu, MS, and Xiaoling Yuan, MD \\ Department of Neurology, Liaocheng People's Hospital, Liaocheng, Shandong, China
}

OBJECTIVE Lumbar puncture may not be suitable for some patients needing subarachnoid puncture, while lateral C1-2 puncture and cisterna magna puncture have safety concerns. This study investigated lateral atlanto-occipital space puncture (also called lateral cisterna magna puncture) in patients who needed subarachnoid puncture for clinical diagnosis or treatment. The purpose of the study was to provide information on the complications and feasibility of this technique and its potential advantages over traditional subarachnoid puncture techniques.

METHODS In total, 1008 lateral atlanto-occipital space puncture procedures performed in 667 patients were retrospectively analyzed. The success rate and complications were also analyzed. All patients were followed up for 1 week after puncture.

RESULTS Of 1008 lateral atlanto-occipital space punctures, 991 succeeded and 17 failed (1.7\%). Fifteen patients $(2.25 \%)$ reported pain in the ipsilateral external auditory canal or deep soft tissue, 32 patients $(4.80 \%)$ had a transient increase in blood pressure, and 1 patient $(0.15 \%)$ had intracranial hypotension after the puncture. These complications resolved fully in all cases. There were no serious complications.

CONCLUSIONS Lateral atlanto-occipital space puncture is a feasible technique of subarachnoid puncture for clinical diagnosis and treatment. It is associated with a lower rate of complications than lateral C1-2 puncture or traditional (suboccipital) cisterna magna puncture. It may have potential in the neurological diagnostic and treatment fields.

https://thejns.org/doi/abs/10.3171/2017.1.JNS161089

KEY WORDS lateral atlanto-occipital space puncture; lateral cisterna magna puncture; spinal puncture; treatment outcome; diagnostic technique; adverse effects

\begin{abstract}
$\mathrm{S}$ UBARACHNOID puncture is a routine technique in both the basic neurosciences and clinical practice, which despite the development of advanced imaging modalities, such as CT, has not been entirely replaced..$^{15}$ The most common method of subarachnoid puncture is lumbar puncture (LP), and this is generally considered to be safe with few complications. ${ }^{22}$ Nevertheless, LP has some contraindications, such as in patients with increased intracranial pressure or lesions in the puncture site. Also, LP may not be feasible in patients who are unable to maintain the required position, either seated or lying on their side. ${ }^{8,21}$ Other methods for subarachnoid puncture such as lateral C1-2 puncture and suboccipital cisterna magna puncture are seldom used because of anatomical issues and risks of major complications. ${ }^{13,16}$
\end{abstract}

To find a safe and convenient method that may be a suitable alternative to LP, Professor Dianrong Gong has, since 1990, steadfastly studied the anatomical characteristics of the atlanto-occipital space and cisterna magna and specific puncture sites and has performed repeated simulated procedures in specimens in the Department of Neurology, Liaocheng People's Hospital. After she had mastered the main points and methods of the puncture procedure, the technique of lateral atlanto-occipital space puncture was applied in the clinic beginning in 1995. As of this writing, we have conducted 1008 lateral atlanto-occipital space punctures for clinical diagnosis and treatment. Since July 2011, 229 patients with refractory neurological disease have received 340 human umbilical cord blood mononuclear cell transplantations via lateral atlanto-occipital 
space puncture, and there have been no cases of iatrogenic injury.

We present a retrospective analysis of a large case series of patients who underwent lateral atlanto-occipital space puncture in our clinic. Our goal is to provide details on the procedure as well as the success and safety of the method in the hope that this information may assist other clinicians who require an alternative to LP for subarachnoid puncture.

\section{Methods \\ Patient Group}

From March 1995 to March 2015, 667 patients hospitalized in the Department of Neurology, Liaocheng People's Hospital, received 1008 lateral atlanto-occipital space puncture procedures for clinical diagnosis or treatment (Table 1). The method was approved by the medical ethics committee of the hospital. Of the 667 patients, 389 (58.32\%) were male and $278(41.68 \%)$ were female. Their ages ranged from 5 to 88 years (median 59 years, mean $[ \pm \mathrm{SD}] 56.78 \pm 18.13$ years). Two hundred twenty-nine of the patients had refractory neurological diseases and were undergoing treatment with human umbilical cord blood mononuclear cell transplantation; these patients were treated between July 2011 and March 2015.

The indications for lateral atlanto-occipital space puncture were as follows: ${ }^{3,21}$ 1) inflammation of the CNS; 2) diagnosis of subarachnoid hemorrhage in patients with negative brain CT or difficult differential diagnosis of meningitis; 3) CSF replacement or intrathecal drug therapy; 4) stem cell transplantation; 5) diagnosis and differential diagnosis of demyelinating disease; 6) treatment of ventricular hemorrhage; 7) alternative to difficult LP; 8) rescue of patients with brain herniation of varying causes; or 9) need for CSF specimen or intrathecal treatment in critically ill patients who were not suitable candidates for LP or radiographic investigation.

Lateral atlanto-occipital puncture was not performed in patients who could not cooperate because of tension or those with any of the following contraindications:3,21 1) local infection of the puncture site; 2) allergic reaction to local anesthetic; 3) critical clinical condition, such as severe cardiopulmonary dysfunction; 4) intracranial spaceoccupying lesions; or 5) severe coagulation disorders.

\section{Data Collection}

The baseline characteristics of the patients were collected from their medical records. These data included their age and sex as well as the reason for the puncture procedure and the clinical diagnosis. The success of the procedure was noted and any reasons for unsuccessful puncture recorded.

\section{The Lateral Atlanto-Occipital Space Puncture Procedure}

Diagrams presenting the location of the puncture site are presented in Figs. 1-3, and a video clip demonstrating the procedure is shown in Video 1.

VIDEO 1. Video clip showing the procedure for lateral atlantooccipital space puncture. Copyright Dianrong Gong. Published with permission. Click here to view.
TABLE 1. Baseline characteristics of the patients who underwent lateral atlanto-occipital space puncture

\begin{tabular}{lc}
\hline \multicolumn{1}{c}{ Characteristic } & Value \\
\hline Age in yrs & \\
\hline Mean \pm SD & $56.78 \pm 18.13$ \\
\hline Median & $5-88$ \\
\hline Range & $389(58.32)$ \\
\hline Sex, no. of pts (\%) & $278(41.68)$ \\
\hline Male & \\
\hline Female & $196(19.44)$ \\
\hline Reason for puncture, no. of punctures (\%) & $812(80.56)$ \\
\hline Diagnosis & $250 / 309$ \\
\hline Treatment & $121 / 186$ \\
\hline Disease, no. of pts/punctures & $19 / 19$ \\
\hline Subarachnoid hemorrhage & $15 / 15$ \\
\hline CNS infection & $8 / 20$ \\
\hline Guillain-Barré syndrome & $17 / 17$ \\
\hline Coma of unknown cause & $1 / 1$ \\
\hline Intrathecal injection of drugs for delayed encepha- \\
$\quad$ lopathy after acute CO poisoning \\
\hline Dredge treatment when the lateral ventricle drain- \\
$\quad$ age tube was not free & $229 / 420$ \\
\hline Whiplash injury syndrome & \\
\hline Cerebellar tonsillar hernia after LP & \\
\hline Chemotherapy w/ intrathecal injection & \\
\hline Umbilical cord blood stem cell therapy & \\
\hline
\end{tabular}

Pts $=$ patients.

The procedure is as follows. The patient is placed in a supine position with any pillow removed (although a pillow could be placed under the shoulders of an obese patient to facilitate the procedure if necessary). The patient is required to keep the head and neck straight and in the midline position. The highest point of the mastoid is located, and the puncture site is identified as $1 \mathrm{~cm}$ inferior and $1 \mathrm{~cm}$ posterior to that point (between the lower edge of the occipital bone and atlas). After local sterilization and induction of local anesthesia by infiltration of 2.5-3 $\mathrm{ml}$ of a solution containing $400 \mathrm{mg}$ lidocaine per $20 \mathrm{ml}$, a 20-gauge needle $(0.9-\mathrm{mm}$ outer diameter, Shanghai SA Medical \& Plastic Instruments Co.) is used to pierce the skin. (The puncture needle consists of 2 parts: one part is the needle with a hollow structure; the other part is the needle core.) The puncture direction is perpendicular to the neck, parallel to the imaginary line of both sides of the external auditory canal, and just below the occipital bone. Viewed from the side, the needle passes through the rear of the atlanto-occipital joint (the vertebral arteries run on both sides of the atlanto-occipital joints), posterior and external to the spinal canal, and between the spinal cord and posterior arch. Viewed from the front, the needle is located above the odontoid and lateral mass (the vertebral artery runs behind the lateral mass). Radiographs of a successful puncture are shown in Fig. 4. No important blood vessels or nerves run very close to the puncture route, so 


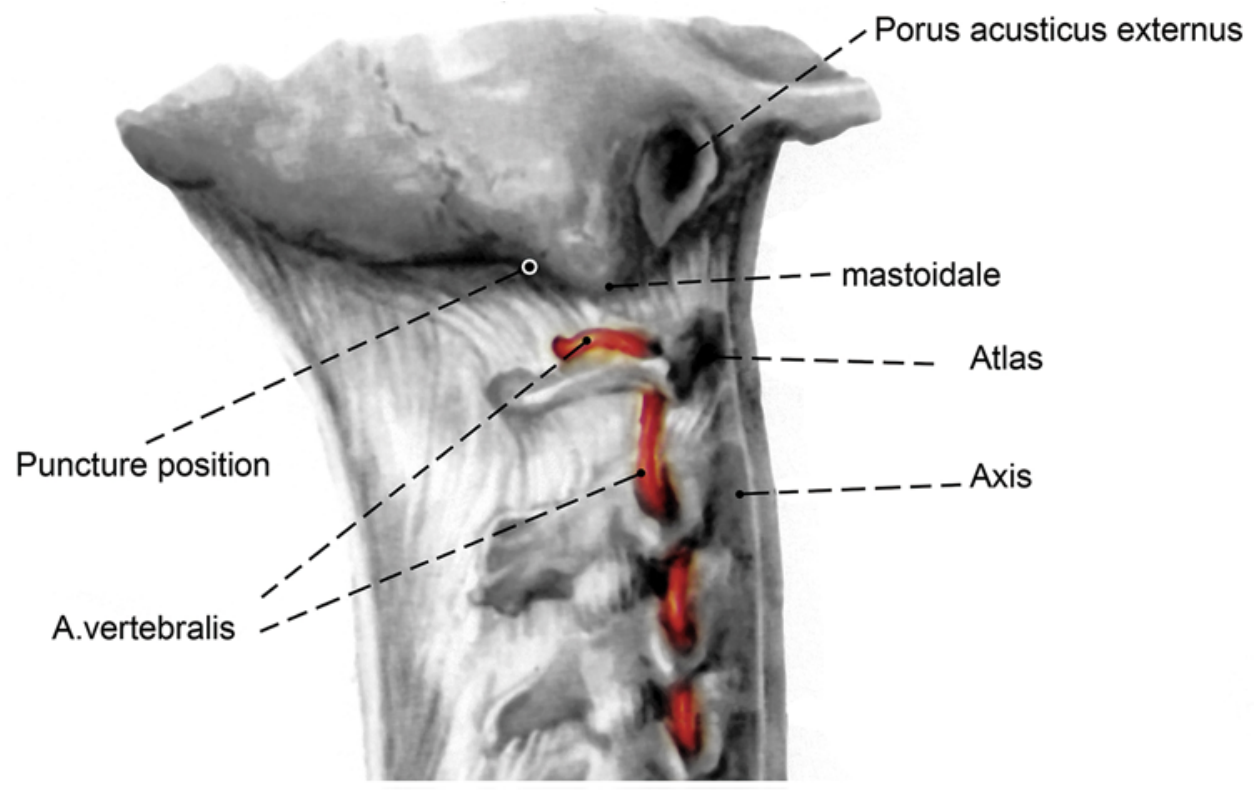

FIG. 1. Sagittal section showing the correct angle of the needle for lateral atlanto-occipital space puncture. $A .=$ arteria. From Guo Guangwen and Wang Xu: Color Atlas of Human Anatomy[M], 2001, page 8. Beijing: People's Medical Publishing House. Published with permission from the authors of the work. Figure is available in color online only.

there is a good safety margin that reduces the risks of the procedure.

Generally, 2 points of membrane breakage can be felt during the course of puncture: the first is perforation of the atlanto-occipital posterior membrane, and the second is perforation of the dura. In general, the puncture depth for an adult is $5.5-6.5 \mathrm{~cm}$. If a depth of more than $6 \mathrm{~cm}$ is reached with no CSF outflow, further insertion of the needle is carried out with great care. The needle core is retracted after 1-2 $\mathrm{mm}$ of insertion to see if there is CSF outflow. The detection of 2 points of membrane breakage can be taken to mean that the needle has entered the cisterna magna, and the puncture is considered successful when CSF is able to flow (see Fig. 5). At this point, according to the clinical requirements of the patient, we either obtain or replace CSF or inject the necessary drugs. The needle core is then removed, and pressure is applied to the puncture point for 1 minute.

\section{Follow-Up and Adverse Events}

Patients were followed up daily for 1 week after puncture. Hospitalized patients were followed up on the ward, while patients who had been discharged from the hospital were followed up at the outpatient department or by telephone. The potential risks and complications of lateral atlanto-occipital space puncture were considered to be the following:,321 1) allergic reaction to the local anesthetic; 2) local infection or bleeding at the puncture site; 3) nicking of an anatomical variant vessel; 4) intracranial hypoten-
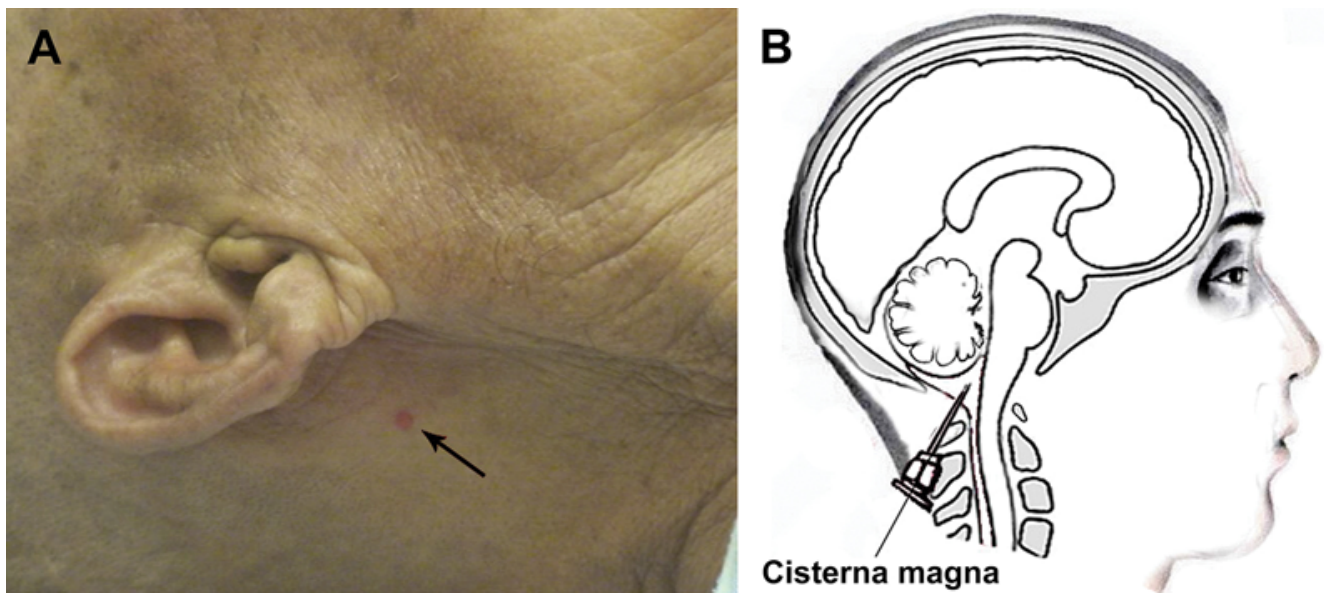

FIG. 2. The puncture point for atlanto-occipital space puncture. A: Location of the puncture site (arrow). B: Schematic illustration showing the needle in the cisterna magna. Figure is available in color online only. 


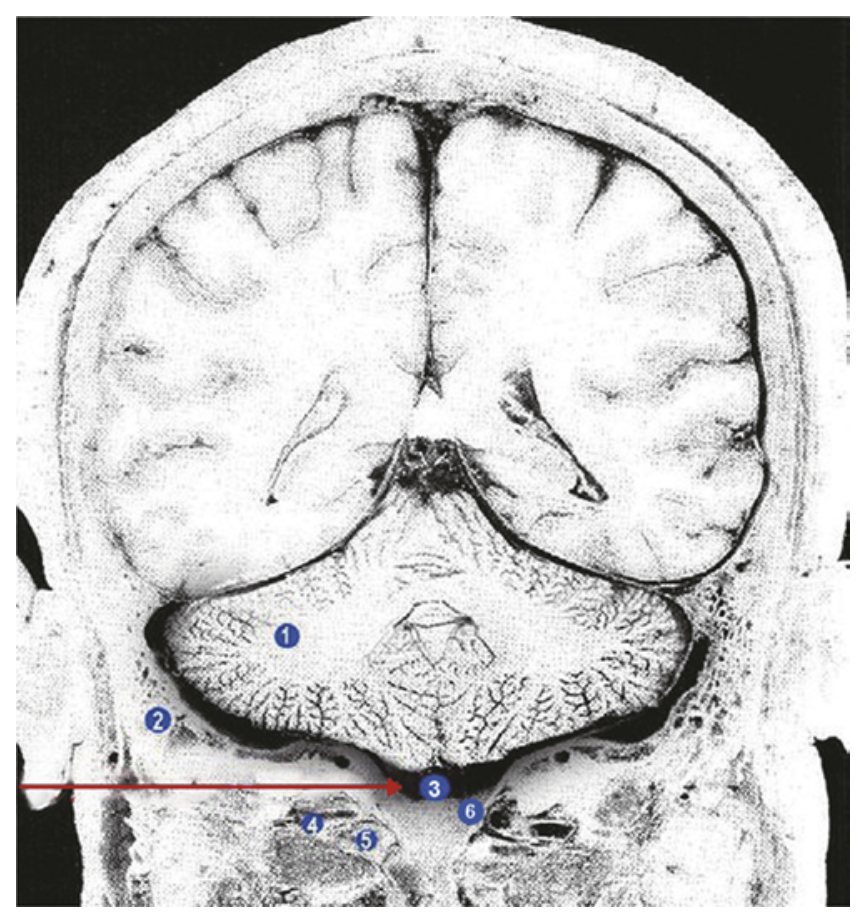

FIG. 3. Coronal section showing the path taken by the needle during lateral atlanto-occipital space puncture (arrow), with anatomical structures numbered as follows: 1 , the cerebellar hemisphere; 2, the occipital bone; 3 , the cisterna magna; 4 , the vertebral artery; 5 , the transverse process of the atlas; and 6, the spinal dura mater. From Liu Shuwei: Atlas of Human Sectional Anatomy[M], 2003, page 41. Jinan City: Shandong Science and Technology Press. Published with permission from the authors of the work. Figure is available in color online only.

sion headache after the puncture; and 5) injury to the neuraxis due to an improper trajectory. The occurrences of any adverse events, including puncture-related local bleeding, infection, fever, or intracranial hypotension headache were recorded.

\section{Statistical Method}

Data were analyzed using SPSS 19.0 (IBM Corp.). The data are presented as means and standard deviations, medians and ranges, and frequencies or percentages.

\section{Results}

\section{Patient Characteristics}

Since 1995, 1008 lateral atlanto-occipital space punctures in 667 patients have been conducted in our hospital. The baseline characteristics of the patients are presented in Table 1 . There was a wide range of reasons for the procedure; $19.44 \%$ of punctures were undertaken for diagnosis and $80.56 \%$ for treatment (Table 1). A total of 96 of the 667 patients included in the study were selected for lateral atlanto-occipital space puncture because traditional LP was contraindicated (due to high intracranial pressure, coma, endotracheal intubation, cerebellar tonsillar herniation, LP site infection, lumbar vertebral deformation and fusion, or a history of lumbar surgery or steel plate implantation); the remaining 571 patients did not have contraindications to traditional LP.
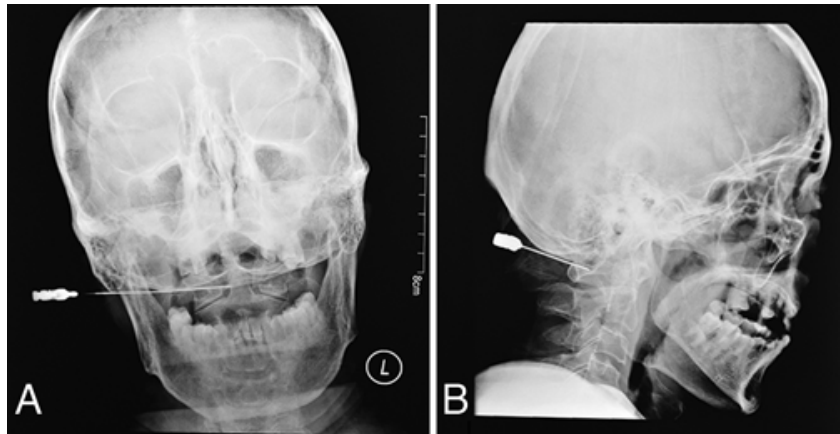

FIG. 4. Anteroposterior $(\mathbf{A})$ and lateral $(B)$ radiographs showing the needle position in lateral atlanto-occipital space puncture.

\section{Success Rate}

The procedure was successful in 991 punctures and unsuccessful in 17. Among the 17 failures, 2 were because the patient would not cooperate during the process and the remaining 15 were due to technical problems. Therefore, the success rate was $98.31 \%$. Detailed analysis of the reasons for failure showed that 11 of the unsuccessful attempts at lateral atlanto-occipital space puncture happened before 2000; we presume that many of these failures resulted from a lack of experience and resulting inaccuracy in the location of the puncture point. Three of the 6 failures that occurred after 2000 involved patients with a spinal deformity that prevented them from lying completely flat, and one involved a patient who found it difficult to adopt the required position because of cerebral palsy (Table 2).

\section{Complications}

The complications experienced by the patients are presented in Table 2. During the puncture process, 15 patients $(2.25 \%)$ reported pain in the ipsilateral external auditory canal or deep soft tissue, with the pain disappearing when the needle was removed. Thirty-two patients $(4.80 \%)$ experienced transient blood pressure elevation, but this was relieved after symptomatic treatment and was considered to be related to mental stress. Only 1 patient $(0.15 \%)$ had intracranial hypotension after the puncture, but this resolved after the patient was given $500 \mathrm{ml}$ of physiological saline by intravenous injection with the instruction to drink more water. There were no reports of local infections or other adverse reactions during or after the puncture process.

\section{Discussion}

This study presents a retrospective case series involving patients who underwent a novel procedure for subarachnoid puncture-the lateral atlanto-occipital space puncture. The data show a high rate of success in a large number of patients and no serious adverse events. Like LP, the lateral atlanto-occipital space puncture, which we refer to as Gong's puncture, is straightforward to perform and does not require fluoroscopic assistance or navigation (although fluoroscopic assistance/navigation might prove useful as an adjunct while learning the technique). Therefore, we suggest that this procedure should be considered as an alternative to LP, especially in patients who have 

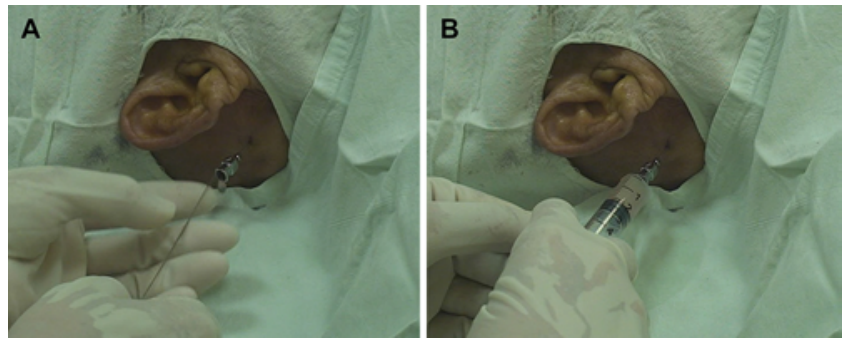

FIG. 5. Successful puncture. A: CSF collection. B: Injection of stem cells. Figure is available in color online only.

contraindications for that technique but who require subarachnoid puncture.

The most common method for subarachnoid puncture is currently LP. ${ }^{1,5}$ Lumbar puncture is a very well established method that shows a high success rate of around 97\%, regardless of whether a blind landmark-guided approach or prepuncture ultrasound assistance is used, ${ }^{4}$ and this method has very few complications. ${ }^{6}$ The most often encountered adverse event with LP is post-dural puncture headache, which occurs in approximately $10 \%-25 \%$ of patients. ${ }^{9,10}$ The success rate (98\%) and safety of lateral atlanto-occipital space puncture observed in this study, with no reports of serious adverse events, are similar to what has been reported for LP.,17 Although the high success rate and low complication rate in our series may be due to the vast experience that we have in performing this technique, we believe that others would be able to rapidly achieve similar success rates due to the simplicity of the technique and the ease with which it could be learned by other clinicians. Furthermore, there may be some advantages of the new method of puncture compared with LP.

With lateral atlanto-occipital space puncture, the patient does not need to be placed in a special position. This makes the procedure especially suitable for patients with serious conditions who cannot be placed in one of the required positions for LP or for patients who have undergone endotracheal intubation or have disturbed consciousness, excessive muscle tone, or lumbar lesions. We have used lateral atlanto-occipital space puncture for diagnosis in patients with severe coma who needed urgent CSF sampling and to rescue patients with herniation caused by LP. (For example, in October 1996, a patient diagnosed with tuberculous meningitis suffered cerebellar tonsillar herniation after LP due to increased intracranial pressure and developed symptoms and signs of bilateral mydriasis, hypotension, respiratory arrest, and decreased heart rate; the patient was successfully treated by lateral atlanto-occipital space puncture and rapid injection of $75 \mathrm{ml}$ of mannitol into the cisterna magna.) In addition, the supine position can reduce or eliminate the patient's fear, so we found that the patients were more willing to accept the examination and treatment. However, while lateral atlanto-occipital space puncture is suitable for patients needing sampling or replacement of CSF or injection of drugs into the spinal canal, the new method cannot replace LP for patients who require gas injection therapy for spinal arachnoid adhesions.

There are also advantages of lateral atlanto-occipital space puncture over lateral C1-2 puncture $^{18,19}$ and the traditional cisterna magna puncture. Because the vertebral
TABLE 2. Success rate and complications experienced with lateral atlanto-occipital space puncture

\begin{tabular}{|c|c|}
\hline Variable & Value \\
\hline \multicolumn{2}{|l|}{ Success of 1st puncture } \\
\hline Successful & $991(98.31 \%)$ \\
\hline Unsuccessful & $17(1.69 \%)$ \\
\hline \multicolumn{2}{|l|}{ Reason for failure } \\
\hline $\begin{array}{l}\text { Puncture point position not accurately identified due } \\
\text { to lack of experience before yr } 2000\end{array}$ & $11(64.71 \%)$ \\
\hline Pt could not lie on back b/c of spinal malformation & $3(17.65 \%)$ \\
\hline Positioning of pt was difficult b/c of cerebral palsy & $1(5.9 \%)$ \\
\hline Pt would not cooperate & $2(11.76 \%)$ \\
\hline \multicolumn{2}{|l|}{ Complications, no. of pts } \\
\hline Pain in ipsilateral EAC or deep soft tissue & 15 \\
\hline Transient BP elevation & 32 \\
\hline Intracranial hypotension & 1 \\
\hline
\end{tabular}

$\mathrm{BP}=$ blood pressure; $\mathrm{EAC}=$ external auditory canal.

Values are no. (\%) of punctures unless otherwise specified.

artery is located with the intervertebral foramen at the level of the C1-2 intervertebral space, and is relatively fixed, it can be easily damaged during lateral $\mathrm{C} 1-2$ puncture. Moreover, in $1 \%$ of cases the vertebral artery runs abnormally, and in these cases a lateral $\mathrm{C} 1-2$ puncture can be fatal. An example of this is provided in a report of a patient with severe meningitis who was given cervical punctures and intrathecal injections; on the sixth occasion the vertebral artery was stabbed, and the patient died after 10 minutes. ${ }^{16}$ Therefore, this method is no longer used clinically. The cisterna magna is located between the cerebellum and medulla oblongata, is formed by the enlarging and expanding subarachnoid space, and connects directly with the fourth ventricle. ${ }^{3}$ The traditional cisterna magna puncture is potentially dangerous because the puncture needle directly faces the medulla oblongata. If the puncture is too deep, it will damage this structure. Because of this risk, cisterna magna puncture is used in animal experiments but seldom in trauma and neurosurgery clinics. ${ }^{7,14}$ We have found, however, that puncture of the cisterna magna via the lateral atlanto-occipital space is safe. The vertebral artery enters the transverse foramen of the C-6 and traverses through each transverse foramen to reach the transverse foramen of C-1 (atlas). It then bends and runs horizontally to the vertebral artery sulcus located behind the lateral mass of the atlas, runs in the middle of this groove, and merges with the contralateral vertebral artery to form the basilar artery at the superoposterior aspect of the spinal cord. During lateral atlanto-occipital space puncture, the needle remains underneath the occipital bone whereas the vertebral artery runs in the sulcus located more posteriorly and medially. It would be difficult to puncture the vertebral artery in cases in which it runs abnormally because of the large amount of surrounding soft tissue and the high range of motion of the vertebral artery at this level. Although vascular studies could potentially be used to identify tortuous or aberrant vertebral artery anatomy, it should be noted that we did not carry out vascular studies before lateral atlanto-occipital space puncture, and despite 
this there were no occurrences of vertebral artery injury during the 1008 procedures performed. We believe that the indications for lateral atlanto-occipital space puncture are numerous and that the procedure can be used in all patients who are suitable for lateral C1-2 puncture.

The new procedure also shows potential for use in stem cell therapy. Some intractable neurological diseases, such as degenerative diseases (Alzheimer's disease, multiple system atrophy, and Parkinson's disease) and delayed encephalopathy after carbon monoxide intoxication, cannot be cured by traditional treatments. In these cases, stem cell transplantation offers some hope. ${ }^{12,20}$ Currently common methods for stem cell transplantation are lateral ventricle puncture and LP. Stem cells can be successfully administered via LP, and this method results in larger numbers of migrated cells than intravenous administration. ${ }^{11}$ However, stem cells administered via LP must pass through the CSF circulation, and this is likely to result in the loss of some stem cells. In contrast, stem cells transplanted via lateral ventricle puncture can migrate specifically to the damaged area of the brain and integrate well with the host cells in terms of morphology and function. ${ }^{2}$ The lateral ventricle puncture site is much closer to the brain, the pathway is shorter, and less stem cell loss occurs compared with LP. Hence, lateral ventricle puncture is an effective method of stem cell transplantation. However, this procedure is both complicated and potentially dangerous, and the patient experiences pain and is at high risk of infection; therefore, it is of limited use in clinical studies. Administration of stem cells via lateral atlanto-occipital space puncture directly into the cisterna magna, involving a shorter migration distance in the CSF circulation, is likely to be more effective. The cisterna magna connects directly with the fourth ventricle, so theoretically, lateral ventricle puncture and lateral atlanto-occipital space puncture would have the same treatment effect. Moreover, lateral atlanto-occipital space puncture is straightforward to carry out and does not involve moving the patient into a special position. This study suggests that lateral atlanto-occipital space puncture also has numerous advantages, such as low risk, a small wound, little pain, and a high success rate, and that stem cell transplantation via lateral atlanto-occipital space puncture would be practical and safe in the clinical situation.

This study has some limitations. The main limitation is that the study is a retrospective case series. However, the large number of procedures (1008) and patients (667) strengthens the findings of our study with regard to the success rate and safety of the technique. Based on our results, randomized controlled studies are merited to directly compare our novel technique with alternative procedures such as LP. As a new location for subarachnoid puncture, the technique will require training and experience to ensure its successful application. We selected the puncture point to be $1 \mathrm{~cm}$ inferior and $1 \mathrm{~cm}$ posterior to the highest point of the mastoid. As the mastoid is not very prominent in some patients, at times it was not easy to choose the puncture site, and this may lead to puncture failure.

\section{Conclusions}

Lateral atlanto-occipital space puncture is safe and easy to perform. This method can be used not only as an alternative or complementary procedure to LP but also as a preferred method in the diagnosis and treatment of specific diseases. In addition, lateral atlanto-occipital space puncture provides a new way to transplant stem cells in patients with refractory neurological diseases.

\section{References}

1. Bonadio W: Pediatric lumbar puncture and cerebrospinal fluid analysis. J Emerg Med 46:141-150, 2014

2. Boon JM, Abrahams PH, Meiring JH, Welch T: Lumbar puncture: anatomical review of a clinical skill. Clin Anat 17:544-553, 2004

3. Chevallier S, Monti M, Michel P, Vollenweider P: [Lumbar puncture.] Rev Med Suisse 4:2312-2314, 2316-2318, 2008 (Fr)

4. Conroy PH, Luyet C, McCartney CJ, McHardy PG: Realtime ultrasound-guided spinal anaesthesia: a prospective observational study of a new approach. Anesthesiol Res Pract 2013:525818, 2013

5. Doherty CM, Forbes RB: Diagnostic lumbar puncture. Ulster Med J 83:93-102, 2014

6. Duits FH, Martinez-Lage P, Paquet C, Engelborghs S, Lleó A, Hausner L, et al: Performance and complications of lumbar puncture in memory clinics: Results of the multicenter lumbar puncture feasibility study. Alzheimers Dement 12:154-163, 2016

7. Duris K, Manaenko A, Suzuki H, Rolland W, Tang J, Zhang JH: Sampling of CSF via the cisterna magna and blood collection via the heart affects brain water content in a rat SAH model. Transl Stroke Res 2:232-237, 2011

8. Gorelick PB, Biller J: Lumbar puncture. Technique, indications, and complications. Postgrad Med 79:257-268, 1986

9. Khlebtovsky A, Weitzen S, Steiner I, Kuritzky A, Djaldetti R, Yust-Katz S: Risk factors for post lumbar puncture headache. Clin Neurol Neurosurg 131:78-81, 2015

10. Lavi R, Rowe JM, Avivi I: Lumbar puncture: it is time to change the needle. Eur Neuro 164:108-113, 2010

11. Lim JY, Jeong CH, Jun JA, Kim SM, Ryu CH, Hou Y, et al: Therapeutic effects of human umbilical cord blood-derived mesenchymal stem cells after intrathecal administration by lumbar puncture in a rat model of cerebral ischemia. Stem Cell Res Ther 2:38, 2011

12. Lopez-Leon M, Reggiani PC, Herenu CB, Goya RG: Regenerative medicine for the aging brain. Enliven J Stem Cell Res Regen Med 1:1-9, 2014

13. Luján Feliu-Pascual A, Garosi L, Dennis R, Platt S: Iatrogenic brainstem injury during cerebellomedullary cistern puncture. Vet Radiol Ultrasound 49:467-471, 2008

14. Mahat MY, Fakrudeen Ali Ahamed N, Chandrasekaran S, Rajagopal S, Narayanan S, Surendran N: An improved method of transcutaneous cisterna magna puncture for cerebrospinal fluid sampling in rats. J Neurosci Methods 211:272-279, 2012

15. Martin SC, Teo MK, Young AM, Godber IM, Mandalia SS, St George EJ, et al: Defending a traditional practice in the modern era: The use of lumbar puncture in the investigation of subarachnoid haemorrhage. Br J Neurosurg 29:799-803, 2015

16. Rogers LA: Acute subdural hematoma and death following lateral cervical spinal puncture. Case report. J Neurosurg 58:284-286, 1983

17. Roos KL: Lumbar puncture. Semin Neurol 23:105-114, 2003

18. Rossitti SL, Balbo RJ: [Lateral cervical puncture for myelography and cerebrospinal fluid collection: technical note.] Arq Neuropsiquiatr 46:397-400, 1988 (Portuguese) 
19. Rossitti SL, Balbo RJ: [Lhermitte's sign during lateral cervical puncture: survey of possible accidents in the lateral $\mathrm{C} 1-$ $\mathrm{C} 2$ puncture and report of 2 cases of spinal cord penetration.] Arq Neuropsiquiatr 48:341-347, 1990 (Portuguese)

20. Santos T, Boto C, Saraiva CM, Bernardino L, Ferreira L: Nanomedicine approaches to modulate neural stem cells in brain repair. Trends Biotechnol 34:437-439, 2016

21. Sempere AP, Berenguer-Ruiz L, Lezcano-Rodas M, MiraBerenguer F, Waez M: [Lumbar puncture: its indications, contraindications, complications and technique.] Rev Neuro 145:433-436, 2007 (Span)

22. Williams J, Lye DC, Umapathi T: Diagnostic lumbar puncture: minimizing complications. Intern Med J 38:587-591, 2008

\section{Disclosures}

The authors report no conflict of interest concerning the materi- als or methods used in this study or the findings specified in this paper.

\section{Author Contributions}

Conception and design: Gong, Yuan. Acquisition of data: Yu. Analysis and interpretation of data: Yu. Drafting the article: Gong. Critically revising the article: Gong, Yuan. Reviewed submitted version of manuscript: Gong. Approved the final version of the manuscript on behalf of all authors: Gong.

\section{Supplemental Information \\ Videos}

Video 1. https://vimeo.com/206421629.

\section{Correspondence}

Dianrong Gong, Department of Neurology, Liaocheng People's Hospital, No. 67, Dongchang West Rd., Liaocheng, Shandong 252000, China. email: 13346256059@163.com. 